Folia Primatol 1996;67:222

\title{
Subject Index Vol 67, 1996
}

Adapids 137 Adult males 152 Alaotran gentle lemur 44 Allometry 15 Alouatta seniculus 193 Ateles paniscus 193 Atelines 193

Biomechanics 1

Callithrixjacchus 52 Cannibalism 52 Canopy height 163 Cercopithecus diana 125 Chamaeleo lateralis 40 Chameleon 40 Chimpanzee 15 Climbing 193 Colobus badius 125 Colour pattern 113 Common marmosets 52 Computer simulation 1 Conspecific threat 152

Diurnal lemur 40 DNA typing 157

Energetic costs 44 Eulemur 157 Evolution 182

Face 182

Feeding behavior 163 Female transfer 169 Food competition 169

Galago moholi 1 Gene flow 113 Gibbons 163 Group composition 169

Hapalemur griseus alaotrensis

44 Home range 125 Hominoids 182, 193 Howling monkeys 193 Humeral head 137

Hybridization 113

Image analysis 182 Inbreeding 52 Infant development 44, 152 Infanticide 44, 52, 152, 169

Intrasexual competition 52 Ivory Coast 125

Lactation 44 Langurs 163 Leaping 1 Lemur catta 40 Lemurs 157 Locomotion 1, 137 Loris

tardigradus 1

Macaques 163

Madagascar 40

Male disappearance 169

Man 15

Maternal protectiveness 152

Micwcebus 157

Mitochondrial DNA 113

Morphome tries 15

Morphometry 137

Multivariate analysis 137

Niche partitioning 163

Orangutans 163

Paternity 157

Polygyny 52

Polymerase chain reaction 157

Polyspecific associations 125

Predation 152

Predator avoidance 125

Presbytis thomasi 169

Procrustes 15

Prosimians 157 
Proximal wrist joint 193

Random amplified

polymorphic DNA 157 Reproductive suppression 52 Ring-tailed lemur 40 River barriers 113 Saguinus 113 Shape 15 Size 15 Skull 182

Social structure 152 Spider monkeys 193 Strepsirhines 137 Suspension 193

Tamarins 113 Thin-plate spline 15

Ulnopisiform joint 193

Vertebrate predation 40

Waser's gas model 125 Weaning 44

222 